\title{
Mathematical Modelling of Strongly Non- Equilibrium Transfer Processes at Nanoscopic Scale
}

\author{
I.V. Kudinov \\ Heat Engineering Theoretical Foundations and \\ Hydromechanics department \\ Samara State Technical University, SSTU \\ Samara, Russian Federation \\ igor-kudinov@bk.ru
}

A.V. Eremin

Heat Engineering Theoretical Foundations and

Hydromechanics department

Samara State Technical University, SSTU

Samara, Russian Federation

a.v.eremin@list.ru

\author{
V.K. Tkachev
}

Heat Engineering Theoretical Foundations and Hydromechanics department

Samara State Technical University, SSTU

Samara, Russian Federation

totig@yandex.ru

\begin{abstract}
Based on the concepts of local non-equilibrium thermodynamics, mathematical models for heat, mass and momentum transfer have been developed with space-time nonlocality taken into consideration. Derivation of transfer differential equations is based on taking into account both specific flows (of heat, mass and momentum) and gradients of corresponding values in diffusion laws by Fourier, Fick and Newton. The research of accurate analytical solutions of the derived models enabled us to find new change patterns of the required parameters for small and ultra small values of time and space variables, as well as for high-speed processes, the change time of which is comparable to relaxation time. Particularly, from the investigation of an accurate analytical solution, it has been found that there is a time delay for Derichlet's boundary condition acceptance, which demonstrates that due to body's resistance to heat penetration, its instant heat-up is impossible, irrespective of any heat exchange with the environment. Therefore, wall heat exchange factor depends not only on heatexchange conditions (medium speed, viscosity, etc.), but also on the body's physical properties. So, firstly, it is a time-variant and, secondly, it can not exceed a certain limit value, established for each particular case. The conducted research of the rod oscillations with relaxation phenomena, taken into consideration, discovered bifurcation oscillations (beat) appearing under the external harmonic load in cases when the difference between the frequency of eigen-oscillations of the rod and constrained load oscillations is insufficient.
\end{abstract}

Keywords - Local-nonequilibrium processes, space-time nonlocality

\author{
V.A. Kudinov \\ Heat Engineering Theoretical Foundations and \\ Hydromechanics department \\ Samara State Technical University, SSTU \\ Samara, Russian Federation \\ kud-samgtu@yandex.ru
}

K.V. Trubitsyn

Heat Engineering Theoretical Foundations and Hydromechanics department

Samara State Technical University, SSTU

Samara, Russian Federation

totig@yandex.ru

E.V. Stefanyuk

Heat Engineering Theoretical Foundations and Hydromechanics department

Samara State Technical University, SSTU

Samara, Russian Federation

stef-kate@yandex.ru

\section{INTRODUCTION}

Recently, due to the necessity of carrying out different physical processes under the extreme conditions, the interest in studying locally non-equilibrium processes has risen. The interest to such processes is stipulated by the possibilities of learning new laws and material properties, which may be widely applied for developing new technologies of nanomaterials and coatings with unique physical and chemical properties. However, to describe processes running in local non-equilibrium conditions, classic non-equilibrium process of thermodynamics may not be applied since it is based on the local equilibrium principle which is fair for relatively slow processes. There is a problem in developing the adequate physical and the respective mathematical models to describe local non-equilibrium processes, based on a recent third-type thermodynamics version - extended irreversible thermodynamics, which is beyond the scope of the local equilibrium principle [ $1-13]$.

Many theories of local non-equilibrium transfer, which often do not correlate with each other, enable us to conclude, that there is no single uniform theory in this research area. Let us also mention that there is practically no theories of local non-equilibrium momentum transfer applied to a vast group of oscillation processes (rods, springs, strings, elastic fluid, propagation of electromagnetic fields, etc.), described by wave equations, in which due to the use of non-relaxation forms of 
Hooke's, Newton's, Ohm's laws for their derivation, the velocity of propagation of field potentials under investigation turns out to be infinite.

\section{FUNDAMENTAL PROVISIONS OF THE PROPOSED RESEARCH METHOD}

Known mathematical statements of boundary problems of mathematical physics are based on infinite propagation velocity of potentials of fields under investigation (thermal conductivity, heat exchange in liquids, dynamic thermoelasticity, vibrations of elastic bodies and liquids and etc.). It constitutes the ground of the respective diffusion equations: Fourier's law (for heat flow), Fick's law (for diffusion problems), Hooke's law (for normal stress), Newton's law (for shearing stress in liquid), Ohm's law (for current propagation in conductors) and etc. Differential equations obtained based on these laws are local, i.e. spatial and temporal non-locality is not considered in them. For example, mathematical statements of classic unstable heat and mass transfer problems are based on parabolic equations, which derivation is based on the heat balance equation (energy conservation law) and phenomenologic Fourier's law for heat flow (Fourier's hypothesis):

$$
q=-\lambda \partial T / \partial x
$$

where $q$ - heat flow; $\lambda$ - heat conductivity factor; $T-$ temperature; $x$ - coordinate.

Researching into analytical solutions of equations obtained in this method allows concluding that heat propagation velocity in the media being studied is infinite. For the most practical unstable thermal conductivity practical problems, classic Fourier's phenomenology results in satisfactory conclusions confirmed by test data. However, there is a large category of problems for which a classic heat propagation model becomes inconsistent.

These are, for example, boundary problems with small and extrasmall temporal and spatial variables, problems for highspeed processes, which time is compatible with the relaxation time and etc. The main reason of non-applicability of the classic model to the mentioned processes consists in the fact that Fourier's law is just an approximate description of the heat conductivity process, not considering the inertial behavior of heat flow and a scalar temperature gradient value. Maxwell, Vernotte, Cattaneo were the first scientists to introduce the inertial component to the transport equation. In particular, they have introduced a formula for heat flow, known as the Maxwell-Cattaneo formula, by means of using the relaxation component, considering heat propagation end velocity in the Fourier law [3-8]:

$$
q=-\lambda \frac{\partial T}{\partial x}-\tau_{1} \frac{\partial q}{\partial t}
$$

where $t$ - time; $\tau_{1}$ - relaxation coefficient.
By substituting the heat balance equation

$$
c \rho \partial T / \partial t=\partial q / \partial x
$$

with (2), a classic hyperbolic heat transfer equation is derived (in electrical engineering, it is known as the telegraph equation), containing the second time derivative from the required function:

$$
\frac{\partial \Theta(\xi, \mathrm{Fo})}{\partial \mathrm{Fo}}+\mathrm{Fo}_{1} \frac{\partial^{2} \Theta(\xi, \mathrm{Fo})}{\partial \mathrm{Fo}^{2}}=\frac{\partial^{2} \Theta(\xi, \mathrm{Fo})}{\partial \xi^{2}},
$$

where $\Theta=\left(T-T_{\text {ст }}\right) /\left(T_{0}-T_{\text {ст }}\right), \quad \mathrm{Fo}=a t / \delta^{2}, \quad \xi=x / \delta$, $\mathrm{Fo}_{1}=a \tau / \delta^{2} ; a-$ temperature conductivity coefficient; $\delta-$ plate thickness; $c$ - heat capacity; $\rho$-density.

Multiple studies of exact analytical solution of equation (4) allowed concluding that it fails to provide a sufficiently adequate description to the studied processes, the same as the parabolic equation (i.e. when $\mathrm{Fo}_{1}=0$ ). And, in particular, solutions of hyperbolic equation (4) result in temperature jumps, which is equivalent to the occurrence of isotherms inside the body and infinite heat flows at the heat wavefront, as well as the emergence of negative temperature in the return wave. These results witness about violating the principle of the maximum value in boundary problems and the energy conservation law.

Cause analysis of the results, obtained from the solution of equation (4), allowed concluding on the necessity to consider the response rate not only for the heat flow (temporal nonlocality), but also the scalar temperature gradient value (spatial non-locality).

Formula (1) of Fourier's law for heat flow obtained in this manner shall be as follows:

$$
q+\tau_{1} \frac{\partial q}{\partial t}+\tau_{2}^{2} \frac{\partial^{2} q}{\partial t^{2}}+\cdots=-\lambda\left(\frac{\partial T}{\partial x}+\tau_{1} \frac{\partial^{2} T}{\partial x \partial t}+\tau_{2}^{2} \frac{\partial^{3} T}{\partial x \partial t^{2}}+\cdots\right)
$$

where $\tau_{1}, \tau_{2}-$ relaxation coefficients.

Similar equations for Newton's law $\tau=\mu \partial \vartheta / \partial y$ and Hooke's law $\sigma=E \partial U / \partial x$ shall be as follows:

$$
\begin{aligned}
& \tau+\tau_{1} \frac{\partial \tau}{\partial t}+\tau_{2}^{2} \frac{\partial^{2} \tau}{\partial t^{2}}+\cdots=\mu\left(\frac{\partial \vartheta}{\partial y}+\tau_{1} \frac{\partial^{2} \vartheta}{\partial y \partial t}+\tau_{2}^{2} \frac{\partial^{3} \vartheta}{\partial y \partial t^{2}}+\cdots\right) \\
& \sigma+\tau_{1} \frac{\partial \sigma}{\partial t}+\tau_{2}^{2} \frac{\partial^{2} \sigma}{\partial t^{2}}+\cdots=E\left(\frac{\partial U}{\partial x}+\tau_{1} \frac{\partial^{2} U}{\partial x \partial t}+\tau_{2}^{2} \frac{\partial^{3} U}{\partial x \partial t^{2}}+\cdots\right)
\end{aligned}
$$

where $\tau, \sigma$ - shearing and normal stress; $\vartheta$ - velocity; $U$ transport; $\mu$-dynamic viscosity; $E$ - elasticity modulus; $\tau_{1}$, 
$\tau_{2}-$ relaxation coefficients of stresses $\tau$ and $\sigma$ as well as velocity and deformation gradients $\partial \vartheta / \partial y$ and $\partial U / \partial x$.

When expressing $q, \tau, \sigma$, limited by two members of the left and right part of the relations (5) - (7), one finds that:

$$
\begin{aligned}
& q=-\lambda \frac{\partial T}{\partial x}-\lambda \tau_{1} \frac{\partial^{2} T}{\partial x \partial t}-\tau_{1} \frac{\partial q}{\partial t} \\
& \tau=\mu \frac{\partial \vartheta}{\partial y}+\mu \tau_{1} \frac{\partial^{2} \vartheta}{\partial y \partial t}-\tau_{1} \frac{\partial \tau}{\partial t} \\
& \sigma=E \frac{\partial U}{\partial x}+E \tau_{1} \frac{\partial^{2} U}{\partial x \partial t}-\tau_{1} \frac{\partial \sigma}{\partial t}
\end{aligned}
$$

Let us note that relations (8) - (10) coincide with the formulas, obtained in the Onsager's differential equation system formulated by A.V. Lykov (based on the hypothesis of the end velocity of mass and heat diffusion) [8]:

$$
J_{i}=L_{i}^{(r)} \frac{\partial J_{i}}{\partial t}+\sum_{k=1}^{N}\left(L_{i k} X_{k}+L_{i k}^{\prime} \frac{\partial X_{k}}{\partial t}\right)
$$

where $J_{i}-$ the substance flow (heat, mass and etc.); $X_{k}$-thermodynamic driving forces (gradients of the respective values); $L_{i}^{(r)} L_{i k}, L_{i k}^{\prime}$ - constant phenomenological transfer coefficients $\left(L_{i k}=L_{k i}\right)$.

If one sets $L=-\lambda ; L^{(r)}=-\tau_{1}, L^{\prime}=-\lambda \tau_{1}, X_{k}=\partial T / \partial x$, than formula (8) is obtained of (11). If one sets $L=\mu$, $L^{\prime}=\mu \tau_{1}, L^{(r)}=-\tau_{1}, X_{k}=\partial \vartheta / \partial x$, equation (11) will become identical to equation (9) [8]. With $L=E, \quad L^{\prime}=E \tau_{2}$; $L^{(r)}=-\tau_{1}$, one obtains formula (10) of (11). Relations (9), (10) fully coincide with the sophisticated models of the viscoelastic body known as Maxwell and Kelvin-Voight models [11]. These models differ in formulas for relaxation coefficient $\tau_{1}$ only. Physical sense of these models lies in the fact that they consider temporal dependence of stresses and deformations and their mutual influence.

\section{Heat Conductivity Mathematical Models CONSIDERING SPATIAL-TEMPORAL NON-LOCALITY}

Let us consider the constructing sequence of local-nonequilibrium heat conductivity models based on formula (5) for heat flow. By substituting (5) in heat balance equation (3), considering three members of the right and left part of relation (5), the heat transfer equation in the non-dimensional form will be as follows $[9,10]$ :

$$
\frac{\partial \Theta}{\partial \mathrm{Fo}}+\mathrm{Fo}_{1} \frac{\partial^{2} \Theta}{\partial \mathrm{Fo}^{2}}+\mathrm{Fo}_{2} \frac{\partial^{3} \Theta}{\partial \mathrm{Fo}^{3}}=\frac{\partial^{2} \Theta}{\partial \xi^{2}}+\mathrm{Fo}_{1} \frac{\partial^{3} \Theta}{\partial \xi^{2} \partial \mathrm{Fo}}+\mathrm{Fo}_{2} \frac{\partial^{4} \Theta}{\partial \xi^{2} \partial \mathrm{Fo}^{2}},
$$

where $\quad \Theta=\frac{T-T_{\mathrm{cT}}}{T_{0}-T_{\mathrm{cT}}} ; \quad \xi=\frac{x}{\delta} ; \quad \mathrm{Fo}=\frac{a t}{\delta^{2}} ; \quad \mathrm{Fo}_{1}=\frac{a \tau_{1}}{\delta^{2}} ;$

$\mathrm{Fo}_{2}=\frac{a^{2} \tau_{2}^{2}}{\delta^{4}}$

If one sets $\mathrm{Fo}_{1}=\mathrm{Fo}_{2}=0$, equation (12) is reduced to a classic parabolic heat conductivity equation.

The exact analytical solution of equation (12) for the infinite plate with the boundary conditions of the first kind shall take the form:

$$
\begin{aligned}
\Theta(\xi, \mathrm{Fo})= & \sum_{k=1}^{\infty}\left\{\left[D_{1 k} \exp \left(z_{1} \mathrm{Fo}\right)+D_{2 k} \exp \left(z_{2} \mathrm{Fo}\right)+D_{3 k} \exp \left(z_{3 k} \mathrm{Fo}\right)\right] .\right. \\
& \left.\cdot \cos \left(r \frac{\pi}{2}(1-2 \xi)\right)\right\}, \quad(r=2 k-1 ; k=\overline{1, \infty})
\end{aligned}
$$

where $z_{1}, z_{2}, z_{3 k}$ - roots of the characteristic equation; $D_{1 k}$, $D_{2 k}, D_{3 k}$ - integration constants, determined based on the initial conditions of the boundary problem; $r=2 k-1$.

Calculation results by formula (14) are given in fig. 1, 2 . Their analysis allows concluding that small values of $\mathrm{Fo}_{1}$ and $\mathrm{Fo}_{2} \quad\left(\mathrm{Fo}_{1}=10^{-6}, \mathrm{Fo}_{2}=10^{-9}\right)$, the obtained solutions within the number range of $10^{-2} \leq \mathrm{Fo}<\infty$ fully coincide with solution of the identical problem for the transient heat conduction parabolic equation.

In case of $\mathrm{Fo}<10^{-2}$, temperature distribution in time has the peculiarities as follows. When $\mathrm{Fo}=10^{-4}$, the temperature of the body in the infinitesimal neighborhood of points $\xi=0$ and $\quad \xi=1$ changes spasmodically from $\Theta\left(0 ; 10^{-4}\right)=\Theta\left(1 ; 10^{-4}\right)=0$ directly at the boundaries (compliance with the first kind boundary conditions (14)) to $\Theta\left(0.0001 ; 10^{-4}\right)=\Theta\left(0.9999 ; 10^{-4}\right)=0.91$ in points $\xi=0.0001$ and $\xi=0.9999$, i.e. at some small distance from points $\xi=0$ and $\xi=1$ (fig. 1).

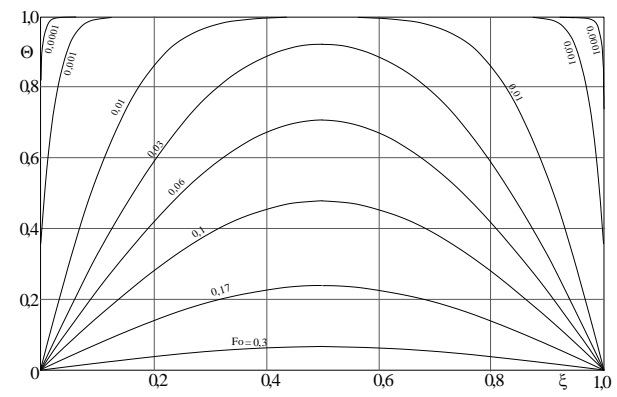

Fig. 1. Temperature distribution in plate. Calculation by formula (14) when $n=100000$, where $n-$ the number of members of series (14). $\mathrm{Fo}_{1}=10^{-3}, \mathrm{Fo}_{2}=10^{-9}$

With the increase of $\mathrm{Fo}_{1}, \mathrm{Fo}_{2} \quad\left(\mathrm{Fo}_{1}=5, \mathrm{Fo}_{2}=10^{-6}\right)$, temperature curves become more flattened and practically parallel to $\xi$. In this case, temperature jump at the wall at 
points $\xi=0$ and $\xi=1$ will take place almost for all the $F_{0}$ numbers up to the development of the steady-state condition (fig. 2).

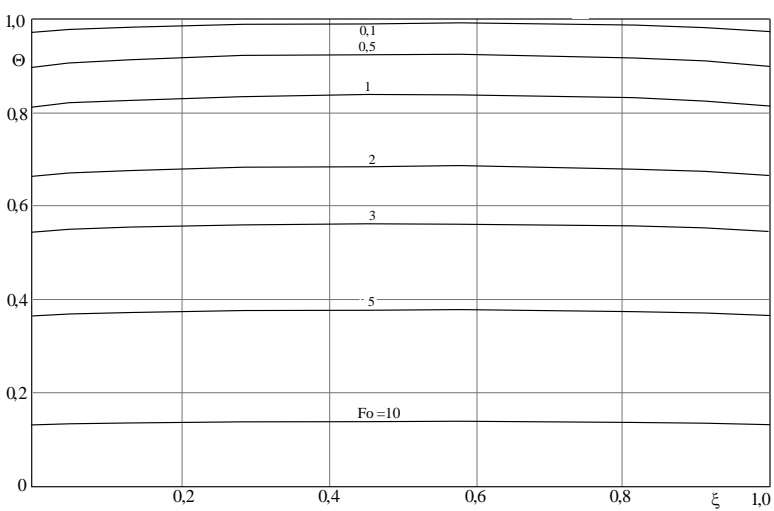

Fig. 2. Temperature distribution when $n=1000 . \mathrm{Fo}_{1}=5, \mathrm{Fo}_{2}=10^{-6}$

Based on the exact analytical solution analysis (14), the fact of time delay in acceptance of the first kind boundary condition established evidences that due to resistance of the body caused to the heat penetration process, its immediate heatup at a boundary is impossible under any conditions of heat exchange with the environment. Consequently, the heattransfer coefficient on the wall depends not only on the heat exchange conditions (medium velocity, viscosity etc.) but also on physical properties of the body. And it is, firstly, a timevariant value, and, secondly, it will not be able to exceed some limiting value for each individual case of the value.

\section{MATHEMATICAL MODEl FOR PRESSURE VARIATION IN INCOMPRESSIBLE FLUID TEMPORAL AND SPECIAL NON- LOCALITY}

Considering relation (9), a differential equation was obtained in work [9] with formulating the boundary problem describing pressure variation in elastic fluid under the hydraulic impact conditions. The mathematical problem definition in this case is as follows:

$$
\begin{gathered}
\mathrm{Fo}_{r} \frac{\partial \Theta(\xi, \mathrm{Fo})}{\partial \mathrm{Fo}}+\frac{\partial^{2} \Theta(\xi, \mathrm{Fo})}{\partial \mathrm{Fo}^{2}}+\mathrm{Fo}_{1} \frac{\partial^{3} \Theta(\xi, \mathrm{Fo})}{\partial \mathrm{Fo}^{3}}= \\
=\frac{\partial^{2} \Theta(\xi, \mathrm{Fo})}{\partial \xi^{2}}+\mathrm{Fo}_{2} \frac{\partial^{3} \Theta(\xi, \mathrm{Fo})}{\partial \xi^{2} \partial \mathrm{Fo}} ; \\
\Theta(\xi, 0)=1 ; \partial \Theta(\xi, 0) / \partial \mathrm{Fo}=0 ; \partial^{2} \Theta(\xi, 0) / \partial \mathrm{Fo}^{2}=0 ; \\
\partial \Theta(1, \mathrm{Fo}) / \partial \xi=0 ; \Theta(1, \mathrm{Fo})=0
\end{gathered}
$$

$$
\text { where } \quad \Theta=\frac{p-p_{1}}{p_{0}-p_{1}} ; \quad \mathrm{Fo}=\frac{c t}{l} ; \quad \xi=\frac{x}{l} ; \quad \mathrm{Fo}_{1}=\frac{c \tau_{1}}{l} \text {; }
$$
$\mathrm{Fo}_{2}=\frac{c \tau_{2}}{l} ; \mathrm{Fo}_{r}=\frac{2 a l}{c} ; \Theta, \mathrm{Fo}, \xi-$ dimensionless pressure, time, coordinate, respectively, $\mathrm{Fo}_{1}, \mathrm{Fo}_{2}-$ dimensionless relaxation coefficients regarding shearing stress and velocity gradient; $\mathrm{Fo}_{r}-$ dimensionless friction factor; $p$ - pressure; $p_{0}-$ initial pressure; $p_{1}-$ pipe inlet pressure; $x$ coordinate; $l$ - pipe length; $c$ - acoustic velocity in fluid; $2 a=32 v / d^{2} ; v$ - fluid viscosity; $d$-pipe diameter.

Boundary problem (15) - (17) describes distribution of the pressure jump in the pipeline with the initial-condition steady fluid for the case when a step pressure change occurred in section $\xi=1$, and section $\xi=0$ is closed (fluid flow velocity is equal to zero). form:

Exact analytical solution of problem (15) - (17) takes the

$$
\begin{gathered}
\Theta(\xi, \mathrm{Fo})=\sum_{k=1}^{\infty}\left[C_{1 k} \exp \left(z_{1 k} \mathrm{Fo}\right)+C_{2 k} \exp \left(z_{2 k} \mathrm{Fo}\right)+C_{3 k} \exp \left(z_{3 k} \mathrm{Fo}\right)\right] \\
\cdot \cos \left(r \frac{\pi}{2} \xi\right)
\end{gathered}
$$

where $C_{1 k}, C_{2 k}, C_{3 k}$ - integration constants determined based on initial conditions (22); $z_{1 k}, z_{2 k}, z_{3 k}$ - roots of the characteristic equation; $(r=2 k-1)$.

Calculation results of formula (18) are given in fig. 3 (for $\xi=0)$

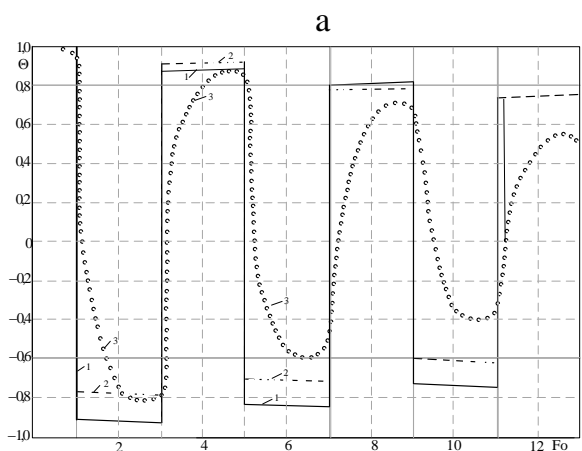

$\mathrm{b}$

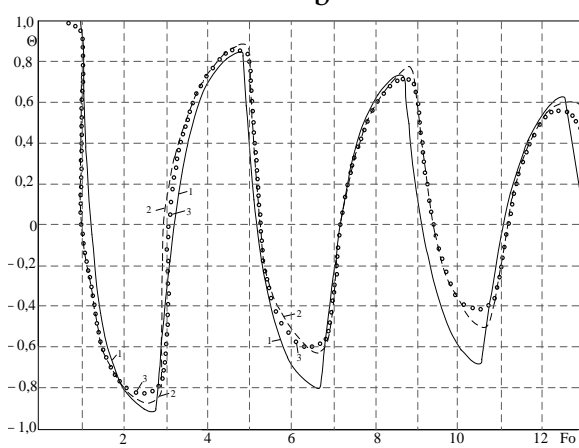

Fig. 3. Pressure distribution under a hydraulic impact $(\xi=0)$ at $\mathrm{Fo}_{r}=0.0538$ and $\mathrm{Fo}_{1}=\mathrm{Fo}_{2}=0$ (a); $\mathrm{Fo}_{r}=0.0538, \mathrm{Fo}_{1}=0.4$, $\mathrm{Fo}_{2}=0.43$ (b): 1 - calculation by formula (18) when $n=100 ; 2$ - using method [12]; 3 - experiment [13] 
Here the results of solving problem (15) - (17) in quasisteady setting are given (with $\mathrm{Fo}_{1}=\mathrm{Fo}_{2}=0$ ) along with the results for a case when the potential propagation end velocity of the studied medium is taken into account $\left(\mathrm{Fo}_{1} \neq 0\right.$, $\mathrm{Fo}_{2} \neq 0$ ), i.e. considering the impact caused by nonstationarity of the process onto the friction force. These figures also represent the results of calculations made by the author of work [12], and the results of experimental studies of the hydraulic impact for a strongly viscous fluid (oil) in a laminar flow regime [12]. Theoretical studies in [12] were performed using two methods: considering the influence of process non-stationarity on the friction force and without considering it (in quasi-static setting).

Based on the experimental data, using relation (18), relaxation coefficients were found by means of solving the inverse problem, which are equal to: $\tau_{1}=0.0108, \mathrm{~s}$, $\tau_{2}=0.0116, \mathrm{~s}$.

\section{Mathematical Model For Heat EXChange IN} FLOWING LIQUID CONSIDERING ITS RELAXATION PROPERTIES

In p. 3, the sequence for obtaining the differential equation of the locally non-equilibrium elastic fluid oscillation process was given. Mathematical model (15) - (17) presented above, motions in a stable fluid under the hydraulic impact conditions were studied. Let us consider derivation of an equation applicable to non-stationary heat exchange at a laminar flow of viscous non-compressible fluid in a plane-parallel channel (energy equation). The formulas for a heat flow along coordinate axes $x$ and $y$, similarly to (8) will be:

$$
\begin{gathered}
q_{x}=-\lambda \frac{\partial T}{\partial x}-\tau_{1} \frac{\partial q_{x}}{\partial t}-\lambda \tau_{2} \frac{\partial^{2} T}{\partial x \partial t}+\rho \omega_{x} i \\
q_{y}=-\lambda \frac{\partial T}{\partial y}-\tau_{1} \frac{\partial q_{y}}{\partial t}-\lambda \tau_{2} \frac{\partial^{2} T}{\partial y \partial t}+\rho \omega_{y} i
\end{gathered}
$$

where $q_{x}, q_{y}$ - heat flows along $x$ and $y ; \omega_{x}, \omega_{y}$ - fluid flow velocity along $x$ and $y ; i$ - heat contents (liquid enthalpy); $x, y$ - longitudinal and transverse coordinates of plane-parallel channel; $\tau_{1}, \tau_{2}$ - relaxation coefficient of temperature gradient and heat flow, respectively.

In this case, heat balance equation will be:

$$
\rho \frac{\partial i}{\partial t}=-\left(\frac{\partial q_{x}}{\partial x}+\frac{\partial q_{y}}{\partial y}\right),(\partial i=c \partial T)
$$

By substituting (20) by (19), neglecting transversal convection heat transfer $\left(\omega_{y} \partial i / \partial y=0\right)$ and longitudinal heat conductivity ( $\lambda \partial^{2} T / \partial x^{2}=0$ ), one obtains (fig. 4):

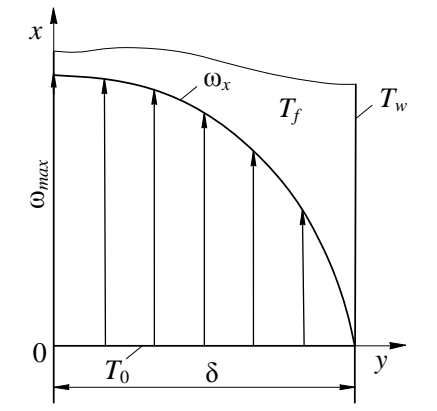

Fig. 4. Heat exchange diagram for laminar flow in plane-parallel channel

$$
\begin{gathered}
\frac{\partial \Theta}{\partial \mathrm{Fo}_{\mathrm{o}}}+\mathrm{Fo}_{1} \frac{\partial^{2} \Theta}{\partial \mathrm{Fo}^{2}}+(1-\eta) \frac{\partial \Theta}{\partial \xi}= \\
=\frac{\partial^{2} \Theta}{\partial \eta^{2}}+\mathrm{Fo}_{2} \frac{\partial}{\partial \mathrm{Fo}}\left(\frac{\partial^{2} \Theta}{\partial \xi^{2}} \mathrm{Pe}+\frac{\partial^{2} \Theta}{\partial \eta^{2}}\right) ;
\end{gathered}
$$

$\Theta(\xi, \eta, 0)=\Theta_{\mathrm{f}} ; \partial \Theta(\xi, \eta, 0) / \partial \mathrm{Fo}=0 ; \Theta(0, \eta, \mathrm{Fo})=1 ;$

$\partial \Theta(0, \eta, \mathrm{Fo}) / \partial \xi=0 ; \partial \Theta(\xi, 0, \mathrm{Fo}) / \partial \eta=0 ; \Theta(\xi, 1, \mathrm{Fo})=0$,

where

$$
\begin{aligned}
\Theta & =\frac{T-T_{\mathrm{w}}}{T_{0}-T_{\mathrm{w}}} ; \quad \eta=\frac{y}{\delta} ; \quad \mathrm{Fo}=\frac{a t}{\delta^{2}} ; \quad \mathrm{Fo}_{1}=\frac{a \tau_{1}}{\delta^{2}} ; \\
\mathrm{Fo}_{2} & =\frac{a \tau_{2}}{\delta^{2}} ; \xi=\frac{2}{3} \frac{a x}{\delta^{2} \omega_{\mathrm{m}}} ; \mathrm{Pe}=\left(\frac{2}{3} \frac{a}{\delta \omega_{\mathrm{m}}}\right)^{2} ;
\end{aligned}
$$

$T_{\mathrm{f}}$ - initial fluid temperature; $T_{0}$ - fluid temperature at the channel inlet; $T_{\mathrm{w}}-$ fluid temperature at the wall; $\delta$ - flat channel width; $\omega_{x}=0.5 \omega_{\mathrm{cp}}\left(1-y^{2} / \delta^{2}\right) ; \omega_{\mathrm{m}}-$ mean velocity of flow. $\Theta_{\mathrm{f}}=\left(T_{\mathrm{f}}-T_{\mathrm{w}}\right) /\left(T_{0}-T_{\mathrm{w}}\right)$.

Fig. 5 represents temperature distribution in fluid at small values of transverse coordinate $0.99 \leq \eta \leq 1.0$ and Fourier number $2 \cdot 10^{-8} \leq \mathrm{Fo} \leq 10^{4}$ obtained by the numerical procedure.

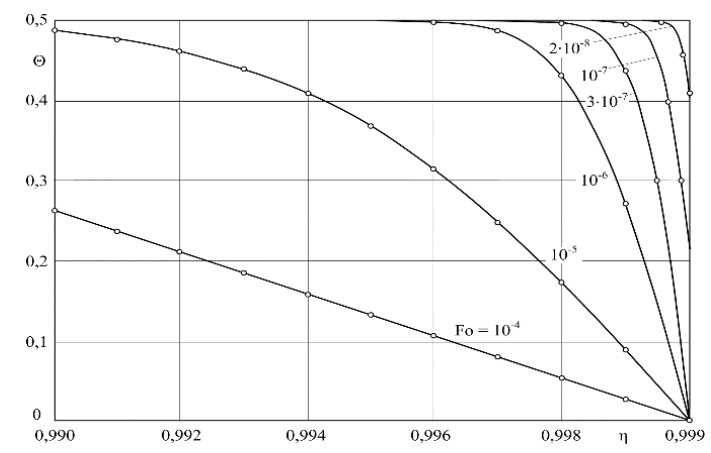

Fig. 5. Temperature distribution along the channel width when $\xi=5 \cdot 10^{-5}$; $\mathrm{Fo}_{1}=\mathrm{Fo}_{2}=10^{-7} ; \mathrm{Pe}=10^{-7}$ within the range of dimensionless time $2 \cdot 10^{-8} \leq \mathrm{Fo} \leq 10^{-4}$. - exact solution [10]; 。- finite difference method 
The analysis of results obtained allows noting an interesting peculiarity of heat exchange when setting the first kind boundary conditions (thermal impact) at the wall in case when the relaxation properties of the medium are considered. Thus, despite complying with the first kind of boundary condition $\Theta(\xi, 1, \mathrm{Fo})=0$ at point $\xi=1$, in the infinitesimal neighborhood of this point, e.g. $\xi=0.999$, the wall temperature does not take on temperature $\Theta(\xi, 1, \mathrm{Fo})=0$ immediately, i.e. directly when $\mathrm{Fo}=0$. And, in particular, some period of time equal to $\mathrm{Fo}=10^{-4}$ (at $\mathrm{Fo}_{1}=\mathrm{Fo}_{2}=10^{-7}$ ) is required for the wall temperature to take on the temperature of $\Theta(\xi, 1, \mathrm{Fo})=0$.

\section{LOCALLY NON-EQUILIBRIUM MODELS OF ELASTIC SOLID BODY OSCILLATION PROCESSES}

Through the use of a modified Hook's law formula in the form of (10), let us consider the constructing sequence for a locally non-equilibrium model for elastic rod oscillation taking into account the resistance caused by the material to the oscillation process. The equilibrium (motion) equation will take on the form:

$$
\rho \frac{\partial^{2} U}{\partial t^{2}}=\frac{\partial \sigma}{\partial x}-\frac{r}{V} \frac{\partial U}{\partial t}
$$

where $\rho$ - density, $\mathrm{kg} / \mathrm{m}^{3} ; \mathrm{r}$ - coefficient, $\mathrm{kg} / \mathrm{s} ; \mathrm{V}-$ volume, $m^{3}$.

Let us note that the second member of sum in the right part of equation (25) represents the resistance force of the medium per unit volume.

By substituting (25) by (11), considering that $\partial \sigma / \partial x=\rho \partial^{2} U / \partial t^{2}$, one finds:

$$
\tau_{1} \frac{\partial^{3} U}{\partial t^{3}}+\frac{\partial^{2} U}{\partial t^{2}}=e^{2} \frac{\partial^{2} U}{\partial x^{2}}+e^{2} \tau_{2} \frac{\partial^{3} U}{\partial x^{2} \partial t}-\gamma \frac{\partial U}{\partial t}
$$

where $\gamma=r /(\rho V)$ - resistance coefficient, $1 / s ; e=\sqrt{E / \rho}-$ wave propagation velocity (sound velocity in the rod material), $\mathrm{m} / \mathrm{s}$.

It is obvious that with $\tau_{1}=\tau_{2}=\gamma=0$, equation (26) will be reduced to the stable oscillation wave equation:

$$
\partial^{2} U / \partial t^{2}=e \partial^{2} U / \partial x^{2}
$$

Let us find a precise analytical solution of the boundary oscillation problem for the rod with one rigidly fixed end and one free end. At the initial time moment, the rod is deformed according to the linear law according to which the maximum motion is characteristic of the free rod end. The mathematical problem setting in dimensionless variable

$$
\begin{array}{r}
\Theta=\frac{U}{U_{0}} ; \quad \xi=\frac{x}{\delta} ; \quad \mathrm{Fo}=\frac{e t}{\delta} ; \quad \mathrm{Fo}_{1}=\frac{e \tau_{1}}{\delta} ; \\
\mathrm{Fo}_{2}=\frac{e \tau_{2}}{\delta} ; \quad \mathrm{Fo}_{3}=\frac{\delta \gamma}{e}
\end{array}
$$

will take the form as follows:

$$
\begin{gathered}
\mathrm{Fo}_{3} \frac{\partial \Theta(\xi, \mathrm{Fo})}{\partial \mathrm{Fo}}+\mathrm{Fo}_{1} \frac{\partial^{3} \Theta(\xi, \mathrm{Fo})}{\partial \mathrm{Fo}^{3}}+\frac{\partial^{2} \Theta(\xi, \mathrm{Fo})}{\partial \mathrm{Fo}^{2}}= \\
=\frac{\partial^{2} \Theta(\xi, \mathrm{Fo})}{\partial \xi^{2}}+\mathrm{Fo}_{2} \frac{\partial^{3} \Theta(\xi, \mathrm{Fo})}{\partial \xi^{2} \partial \mathrm{Fo}} ; \\
\Theta(\xi, 0)=1 ; \frac{\partial \Theta(\xi, 0)}{\partial \mathrm{Fo}}=0 ; \frac{\partial^{2} \Theta(\xi, 0)}{\partial \mathrm{Fo}^{2}}=0 ; \\
\frac{\partial \Theta(0, \mathrm{Fo})}{\partial \xi}=0 ; \Theta(1, \mathrm{Fo})=0,
\end{gathered}
$$

where $\Theta-$ dimensionless motion; $\xi$ - dimensionless coordinate; Fo - Fourier number (dimensionless time); $U_{0}=b \delta ; \mathrm{Fo}_{1}, \mathrm{Fo}_{2}-$ dimensionless relaxation coefficients; $\mathrm{Fo}_{3}$ - dimensionless friction coefficient; $\delta$ - rod length.

The exact analytical solution of problem (29) - (31), found by the variable separation method shall be as follows:

$$
\begin{aligned}
\Theta(\xi, \mathrm{Fo}) & =\sum_{k=1}^{\infty}\left\{\exp \left(\gamma_{k} \mathrm{Fo}\right)\left[B_{1 k} \cos \left(\beta_{k} \mathrm{Fo}\right)-B_{2 k} \sin \left(\beta_{k} \mathrm{Fo}\right)\right]+\right. \\
& \left.+B_{3 k} \exp \left(z_{3 k} \mathrm{Fo}\right)\right\} \cos (r \pi \xi / 2)
\end{aligned}
$$

where $\gamma_{k}, \beta_{k}, z_{3 k}$ - roots of the characteristic equation; $B_{1 k}$, $B_{2 k}, B_{3 k}$ - integration constants, determined based on source conditions (30).

It may be concluded based on the result analysis of calculation by formula (32) that with the increase of $\mathrm{Fo}_{3}$ (with constant $\mathrm{Fo}_{1}$ and $\mathrm{Fo}_{2}$ ), oscillations are displaced to the positive value area of motions and with some higher values of variable $\mathrm{Fo}_{3} \quad\left(\mathrm{Fo}_{3}>5\right)$, the rod returns to the initial condition practically without any oscillations (critical damping) (fig. 6, 7).

Fig. 7 represents calculation results for motions according to formula (32) with $\mathrm{Fo}_{1}=\mathrm{Fo}_{2}=10 ; \mathrm{Fo}_{3}=0$. Their analysis allows concluding that with the increase of $\mathrm{Fo}_{1}$ and $\mathrm{Fo}_{2}$ (when $\mathrm{Fo}_{3}=0$ ) the oscillation amplitude will decrease. Furthermore, multiple oscillation processes occur within the positive motion region only at each point of the rod until its return to the initial (non-deformed) condition. As the time 
increases ( $\mathrm{Fo} \geq 35$ ), the oscillation process becomes stabilized near the zero motion value.
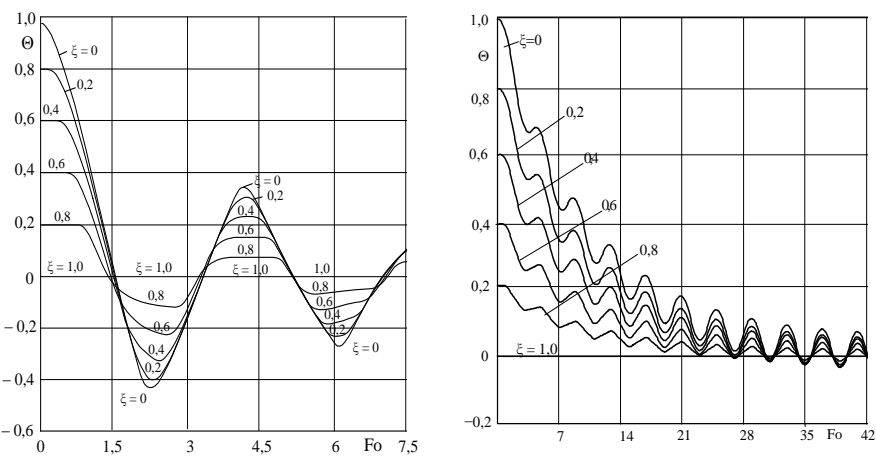

Fig. 6. Motion change $\mathrm{Fo}_{1}=\mathrm{Fo}_{2}=0.1 ; \mathrm{Fo}_{3}=0.5$. $n=100-$ number of series members (32)

\section{EXPERIMENTAL ROD OSCILLATION STUDIES}

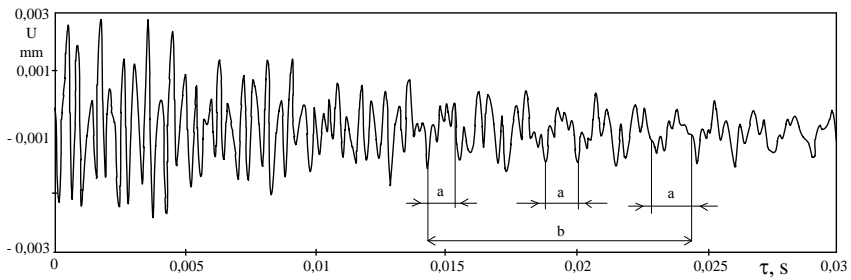

Fig. 8. Rod free end motion

Experimental studies of longitudinal rod oscillation were performed through the use of a special bench. During testing, a $30 \mathrm{~cm}$ long rod fixed on one end was stretched out by $0.1 \mathrm{~m}$. After the load relief, the free end oscillations in time were recorded using a special measuring system. The study results are given in fig. 8. Their analysis allows concluding that the end section of the rod participates in two oscillatory processes - with low amplitude and high frequency (time section $a$ in fig. 8) and simultaneously - with high amplitude and low frequency (time section $b$ in fig. 8).

The obtained experimental data are completely qualitatively in line with the results of theoretic studies according to the elastic body oscillation models, developed by the authors considering special and time non-locality.

\section{RESONANCE ROD OSCILLATION CONSIDERING THE MATERIAL RELAXATION PROPERTIES}

If within problem (29) - (31), the load, changing according to the harmonic law, is applied to the rod free end, the first boundary condition of (31) will be:

$$
\partial \Theta(0, \mathrm{Fo}) / \partial \xi=\mathrm{Fo}_{4} \cos \left(\mathrm{Fo}_{5} \mathrm{Fo}\right),
$$

where $\quad \mathrm{Fo}_{4}=\delta / U_{0} ; \quad \mathrm{Fo}_{5}=\omega \delta / e ; \quad \omega=2 \pi v \quad-$ circular frequency, $1 / s ; v-$ load oscillation frequency, $1 / s$.

Further it will be demonstrated that when the own oscillation frequency coincides with outer load frequency $\mathrm{Fo}_{5}$, resonance oscillations of the rod are observed including bifurcate (flutter) oscillation.

Exact analytical solution of boundary problem (29) - (31), considering the boundary condition (33), becomes considerably complicated; a finite-difference method was used for solving it.

Calculation results for resonance oscillations for $\mathrm{Fo}_{1}=\mathrm{Fo}_{2}=2 ; \mathrm{Fo}_{3}=1 ; \mathrm{Fo}_{4}=0.1 ; \mathrm{Fo}_{5}=1.575$ are given in fig. 9. With the increased relaxation coefficients $\left(\mathrm{Fo}_{1}=\mathrm{Fo}_{2}=10 ; \mathrm{Fo}_{3}=0.3 ; \mathrm{Fo}_{4}=0.1 ; \mathrm{Fo}_{5}=1.575\right)$ during oscillations, bifurcate resonance is observed at which the oscillation amplitude increases periodically, Furthermore, depending on relation of values $\mathrm{Fo}_{1}, \mathrm{Fo}_{2}$ and $\mathrm{Fo}_{3}$, the oscillation process may be both damping and non-damping.

It should be noted that bifurcate change of the oscillation amplitude is also observed at the outer load oscillation frequencies, which are close to own frequency oscillations of the road but not coinciding with it completely. The calculation results for such oscillation type are given in fig. 10, 11 .

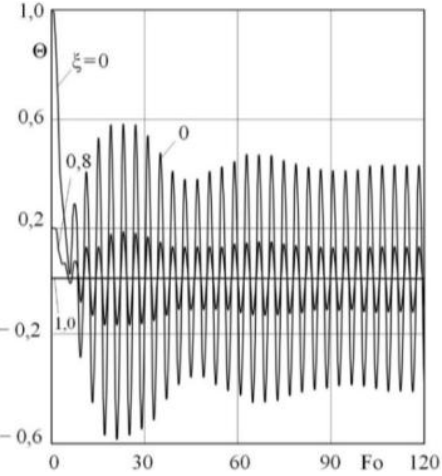

Fig. 9. Resonance frequency change of rod motion when $\mathrm{Fo}_{1}=\mathrm{Fo}_{2}=2$; $\begin{array}{ll}\mathrm{Fo}_{3} & =1 ; \\ \mathrm{Fo}_{5} & =1.575\end{array}$

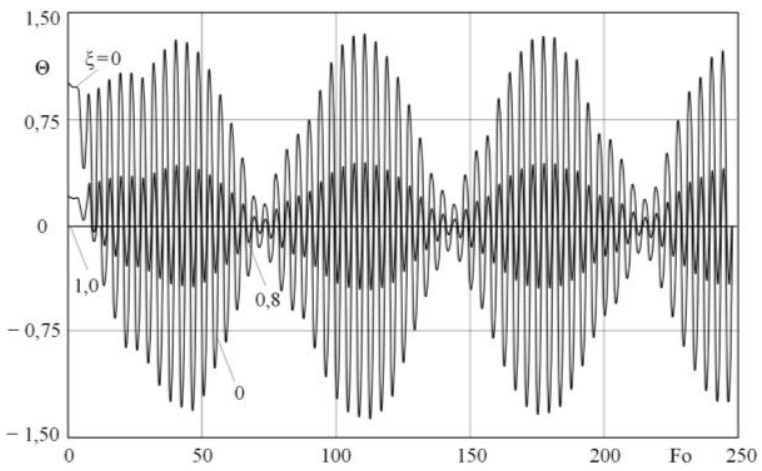

Fig. 11. Non-resonance frequency change of rod motion when $\mathrm{Fo}_{1}=\mathrm{Fo}_{2}=10 ; \mathrm{Fo}_{3}=0.3 ; \mathrm{Fo}_{4}=0.1 ; \mathrm{Fo}_{5}=1.5$

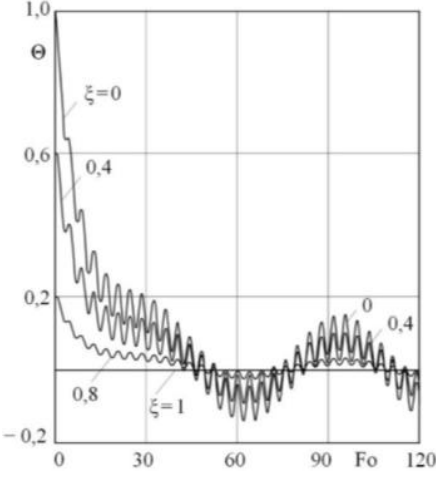

Fig. 10. Non-resonance frequency change of rod motion when $\mathrm{Fo}_{1}=\mathrm{Fo}_{2}=10 ; \quad \mathrm{Fo}_{3}=0.3$ $\mathrm{Fo}_{4}=0.1$ 


\section{CONCLUSION}

On the basis of relaxation equations for the Fourier's law (for the heat flow), Hook's law (for the oscillation theory and dynamic thermoelasticity), Newton's law (tangential stress in fluid), new mathematical models, based on hyperbolic equations, were developed, which describe the distribution of desired potential fields with the finite velocity of their propagation taken into consideration. The expression for a heat flow, which is the basis for deriving the specified hyperbolic equations, is the same as the expression determined from the generalized Onsager's system of equations, obtained by A.V. Lykov based on the hypothesis of the finite heat and mass diffusion velocity. The investigation of the found exact analytical solutions of hyperbolic equations enable us to conclude that there is thermal discontinuity around the boundary spatial coordinate (where a Derichlet's boundary condition is set), which demonstrates physical impossibility of instantaneous warming-up (cooling) of the ambient temperature, regardless of the conditions of external heat exchange.

A model for elastic oscillations of incompressible fluid was developed, which takes into consideration the finite velocity of potential propagation based on estimation of causeand-effect relationship between displacements and stresses in Hook's law formula. The results of theoretical surveys of the oscillations of the rod, fixed at one of its ends, were compared with full-scale experiment data. The results of experimental studies confirmed the results of exact analytical solution, according to which each point of the rod participates in two oscillation processes: one - in high-frequency and lowamplitude oscillations, another one - in low-frequency and high-amplitude oscillations. Using the obtained exact analytical solution and experimental data and by solving an inverse problem, stress and displacement gradient relaxation coefficients were found.

An exact analytical solution of the boundary hydrodynamic problem was obtained, which describes temporally velocity distribution along the pipeline applied to the real viscous incompressible fluid flow under hydraulic shock conditions. The results of theoretical investigations with experimental data were compared for the fluid (oil) under hydraulic shock conditions. Using an exact analytical solution and results of experiments, carried out by E.L. Holmboe and V.T. Rulo, by solving an inverse problem, relaxation coefficients of tangential stress and displacement gradient were found, which turned out to be $\tau_{1}=0.0108 \mathrm{~s}$; $\tau_{2}=0.0116 \mathrm{~s}$. Satisfactory agreement of the results of theoretical and experimental surveys along with the relaxation coefficients, derived this way for both the rod and the incompressible fluid, demonstrates physical legitimacy of representation of Hook's and Newton's laws with timeacceleration of stresses (normal and tangential) and gradients of corresponding values (displacements and velocity) taken into account.

\section{ACKNOWLEDGMENTS}

The study was carried out with the support of the Russian Foundation for Basic Research within the framework of research project No. 16-38-00059 mol_a.

\section{References}

[1] S. De Groote, P. Mazur, Non-Equilibrium Thermodynamics. Moscow: Mir, 1964, pp. $282-311$.

[2] I. Gyarmati, Non-Equilibrium Thermodynamics. Moscow: Mir, 1974, pp. $142-261$.

[3] S.L. Sobolev, "Transfer Process And Progressing Waves In Locally Non-Equilibrium Systems", Success of Physical Science, vol. 161, no. 3, pp. $5-29,1991$.

[4] S.L. Sobolev, "Locally Non-Equilibrium Models Of Transfer Processes", Success of Physical Science, vol. 167, no. 10, pp. $1096-$ 1106, 1997.

[5] D. Jou, J. Casas-Vázquez, G. Lebon, Extended Irreversible Thermodynamics. Moscow - Izhevsk: RDE Regular and Chaotic Dynamics: The Computer Research Institute, 2006, pp. 23 - 90.

[6] A.V. Lykov, Heat Conductivity Theory. Moscow: Vyshaya shkola, 1967, pp. $11-24$.

[7] A.V. Lykov, Heat-Mass Exchange. Reference Book. Moscow: Energiya, 1971 , pp. $526-540$.

[8] A.V. Lykov, "Applying Irreversible Thermodynamics Methods to Heat and Mass Exchange Research", The Engineering and Physical Journal, vol. 9 , no. 3, pp. $287-304,1956$.

[9] I.V. Kudinov, V.A. Kudinov, "Mathematical Simulation of the Locally Nonequilibrium Heat Transfer in a Body with Account for its Nonlocality in Space and Time", Journal of Engineering Physics and Thermophysics, vol. 88, no. 2, pp. $406-422,2015$.

[10] I.V. Kudinov, V.A. Kudinov, Analytical solutions for Parabolic and Hyperbolic Heat And Mass Transfer Equations. Moscow: INFRA - M, 2013, pp. $304-371$.

[11] A.P. Filin, Applied Mechanics Of Solid Deformable Body, vol. 1. Moscow: Nauka, 1975, pp. $751-767$.

[12] I.A. Charnyi, Non-Steady Motion Of Actual Fluid In Pipes. Moscow: Nedra, 1975, pp. $9-22$.

[13] E.L. Kholmbou, V.T. Rulo, "The Impact of Viscous Friction on Propagation of Signals In Hydraulic Lines", Theoretical Fundamentals of Engineering Calculations, no. 3, pp. 203 - 209, 1964. 